\title{
Non-saturation of the $\mathbf{J} / \psi$ suppression at large transverse energy in the comovers approach
}

\author{
A. Capella and E. G. Ferreiro \\ Laboratoire de Physique Théoriquキ \\ Université de Paris-Sud, Bâtiment 210, F-91405 Orsay Cedex, France
}

A. B. Kaidalov

ITEP, B. Cheremushkinskaya, 25, 117259 Moscow, Russia

\begin{abstract}
We show that, contrary to recent claims, the $J / \psi$ suppression resulting from its interaction with comovers does not saturate at large transverse energy $E_{T}$. On the contrary, it shows a characteristic structure - change of curvature near the knee of the $E_{T}$ distribution - which is due to the $E_{T}$ (or multiplicity) fluctuation, and agrees with recent experimental results.
\end{abstract}

LPTHE Orsay 00-21

February 2000

*Unité Mixte de Recherche - CNRS - UMR N 8627 
An interesting result of the last run (1998 data) of the NA50 collaboration at CERN on the transverse energy $\left(E_{T}\right)$ dependence of $J / \psi$ suppression in $\mathrm{PbPb}$ collisions, is the observation [1] of a convexity at large $E_{T}$. More precisely, for $E_{T} \gtrsim 100 \mathrm{GeV}$ (which corresponds to the so-called knee of the $E_{T}$ distribution [1]), the slope of the ratio $R\left(E_{T}\right)$ of $J / \psi$ over Drell-Yan (DY) cross-sections increases with increasing $E_{T}$. In sharp contrast with this result, models of the $J / \psi$ suppression in non-quark-gluon plasma (QGP) scenarios [2-9] - such as the one based on the interaction of the $J / \psi$ with comovers - exhibit a clear saturation at large $E_{T}$.

In this work, we show that the above feature of the comovers model is only true up to the knee of the $E_{T}$ distribution $\left(E_{T} \sim 100 \mathrm{GeV}\right)$. Beyond this value, we enter into the tail of the $E_{T}$ distribution - where the increase in $E_{T}$ is due to fluctuations. This fluctuation, which has not been taken into account in most calculations, produces a corresponding increase in the density of comovers - which, in turn, increases the $J / \psi$ suppression at large $E_{T}$.

In order to illustrate this phenomenon we use the model introduced in ref. [2]. Here, as in most non-QGP models, the $J / \psi$ suppression is due to two mechanisms : absorption of the pre-resonant $c \bar{c}$ pair with nucleons (the so-called nuclear absorption) and the interaction of the $J / \psi$ with comovers. The corresponding $J / \psi$ survival probabilities are given by [2].

$$
\begin{gathered}
S^{a b s}(b, s)=\frac{\left\{1-\exp \left[-A T_{A}(s) \sigma_{a b s}\right]\right\}\left\{1-\exp \left[-B T_{B}(b-s) \sigma_{a b s}\right]\right\}}{\sigma_{a b s}^{2} A B T_{A}(s) T_{B}(b-s)}, \\
S^{c o}(b, s)=\exp \left\{-\sigma_{c o} N_{y}^{c o}(b, s) \ln \left(\frac{N_{y}^{c o}(b, s)}{N_{f}}\right)\right\} .
\end{gathered}
$$

The survival probability $S^{c o}$ depends on the density of comovers $N_{y}^{c o}(b, s)$ in the rapidity 
region of the dimuon trigger $2.9<y_{l a b}<3.9$ and $N_{f}=1.15 \mathrm{fm}^{-2}$ [2, 国 is the corresponding density in $p p$ collisions. In order to compute $N_{y}^{c o}$, various hadronic models have been used in the literature. For instance in ref. [4] it has been assumed that the hadronic multiplicity is proportional to the number of participant nucleons (the so-called wounded nucleon model), while in ref. 22] a formula based on the dual parton model (DPM) was used - which includes an extra term proportional to the number of binary interactions. In this paper we use the DPM formula (eq. (6) of [2]). In both cases, the calculations do not include the fluctuations mentioned above and, therefore, cannot be applied beyond the knee of the $E_{T}$ distribution - where the increase in $E_{T}$ (or multiplicity) is entirely due to fluctuations. In order to introduce these fluctuations, it is convenient to recall the other formulae needed to calculate the $J / \psi$ suppression.

At fixed impact parameter $b$, the $J / \psi$ cross-section is given by [2]

$$
\sigma_{A B}^{\psi}(b)=\frac{\sigma_{p p}^{\psi}}{\sigma_{p p}} \int d^{2} s m(b, s) S^{a b s}(b, s) S^{c o}(b, s),
$$

where $m(b, s)=A B \sigma_{p p} T_{A}(s) T_{B}(b-s)$. The corresponding one for DY pair production is obtained from (3) putting $\sigma_{a b s}=\sigma_{c o}=0$ (i.e. $S^{a b s}=S^{c o}=1$ ) and is proportional to $A B$. In this way we can compute the ratio of $J / \psi$ over DY as a function of the impact parameter. Experimentally, however, the ratio $R\left(E_{T}\right)$, is given as a function of the transverse energy $E_{T}$ measured by a calorimeter, in the rapidity interval $1.1<y_{\text {lab }}<2.3$. In order to compute $R\left(E_{T}\right)$ we have to know the correlation $P\left(E_{T}, b\right)$ between $E_{T}$ and impact parameter, which is given by [2]

$$
P\left(E_{T}, b\right)=\frac{1}{\sqrt{2 \pi q a E_{T}^{N F}(b)}} \exp \left[-\frac{E_{T}-E_{T}^{N F}(b)}{2 q a E_{T}^{N F}(b)}\right]^{2} .
$$

Here 


$$
E_{T}^{N F}(b)=q N_{c a l}^{c o}(b)+k\left[A-m_{A}(b)\right] E_{i n},
$$

$m_{A}(b)$ is the number of participants of $A$ (at fixed impact parameter), $E_{\text {in }}=158 \mathrm{GeV} / \mathrm{c}$ is the beam energy and $k=1 / 4000$ [2]. In (44) and (5) $N_{c a l}^{c o}(b)$ is obtained by integrating the comover density $N_{y}^{c o}(b, s)$ over $d^{2} s$, and $d y$ (in the rapidity range of the $E_{T}$ calorimeter). The second term in (5) was introduced in ref. [2] in order to reproduce the correlation between $E_{T}$ and the energy $E_{Z D C}$ of the zero degree calorimeter. It was interpreted as due to intra-nuclear cascade - which is present here due to the location in rapidity of the NA50 calorimeter. This term is sizable for peripheral collisions, when many spectator nucleons are present, and dies away for central ones. The parameters $q=0.56$ and $a=0.94$ are obtained from a fit to the minimum bias $E_{T}$ distribution at large $E_{T}$. The parameter $q$ gives the relation between multiplicity of comovers (positive, negative and neutrals) and the $E_{T}$ of the NA50 calorimeter (which contains only neutrals). The product qa controls the width of the $E_{T}$ distribution at fixed $b \sim 0$. The $J / \psi$ and DY cross-section at fixed $E_{T}$ are then given by

$$
\frac{d \sigma^{\psi(D Y)}}{d E_{T}}=\int d^{2} b \sigma_{A B}^{\psi(D Y)} P\left(E_{T}, b\right) .
$$

The quantity $E_{T}^{N F}(b)$ in eq. (5) does not contain fluctuations - hence the index $N F$. This is obvious from the fact that the parameter $a$ is not present in (6). In order to see it in a more explicit way, we plot in Fig. 1 the quantity

$$
F\left(E_{T}\right)=E_{T} / E_{T}^{N F}\left(E_{T}\right),
$$

where 


$$
E_{T}^{N F}\left(E_{T}\right)=\frac{\int d^{2} b E_{T}^{N F}(b) P\left(E_{T}, b\right)}{\int d^{2} b P\left(E_{T}, b\right)}
$$

We see that $E_{T}^{N F}$ coincides with $E_{T}$ only up to the knee of the $E_{T}$ distribution. Beyond it, $E_{T}^{N F}$ is smaller than the true value of $E_{T}$. This difference is precisely due to the $E_{T}$ fluctuation.

As discussed above, in order to compute the ratio $R\left(E_{T}\right)$ beyond the knee of the $E_{T}$ distribution it is necessary to introduce in $N_{y}^{c o}$ the $E_{T}$ (or multiplicity) fluctuations responsible for the tail of the distribution. In order to do so, we use the experimental observation that multiplity and $E_{T}$ distributions have approximately the same shape. This indicates that the fluctuations in $E_{T}$ are mainly due to fluctuations in multiplicity rather than in $p_{T}$. This leads to the following replacement in eq. (2)):

$$
N_{y}^{c o}(b, s) \rightarrow N_{y}^{c o}(b, s) F\left(E_{T}\right)
$$

In this way the results for the ratio $R\left(E_{T}\right)$ are unchanged below the knee of the distribution (see Fig. 1). Beyond it, the $J / \psi$ suppression is increased as a result of the fluctuation.

We turn next to the numerical results. In ref. [2] we used for the two parameters of the model $\sigma_{a b s}=6.7 \mathrm{mb}$ and $\sigma_{c o}=0.6 \mathrm{mb}$. In this case, the computed $J / \psi$ suppression at $E_{T} \sim 100 \mathrm{GeV}$ is somewhat too small [2]. Clearly, we can increase it by increasing the value of $\sigma_{c o}$. However, we then increase the value of the suppression for peripheral collisions. This, in turn, can lead to some conflict with the SU data (see [3] for a discussion on this point). However, recent data [10 on the $J / \psi$ cross-section in $p A$ collisions, point to a smaller value of $\sigma_{a b s}$ - of 4 to $5 \mathrm{mb}$. With this value of $\sigma_{a b s}$, we can increase $\sigma_{c o}$ from $0.6 \mathrm{mb}$ up to $1.0 \mathrm{mb}$ without decreasing the $J / \psi$ suppression for peripheral collisions. In Fig. 2 we present the result of our calculation using $\sigma_{a b s}=4.5 \mathrm{mb}$ and $\sigma_{c o}=1 \mathrm{mb}$. We see 
that the main features of the data are reproduced. In particular our curve shows a slight change of curvature at $E_{T} \sim 100 \mathrm{GeV}$, which is entirely due to the effect of fluctuations and is seen in the 1998 NA50 data [1]. The physical origin of this change in the slope of $E_{T}$ is the following: when approaching the knee of the $E_{T}$ distribution from below, the number of participants approaches $2 A$ and changes slowly. The latter is also true for the multiplicity of comovers. Beyond the knee, the multiplicity increases faster due to the fluctuations and produces a faster decrease of $R\left(E_{T}\right)$ (see Fig. 1).

We want to stress that the shape of our curve in the lower half of the $E_{T}$ region (where the ratio $R\left(E_{T}\right)$ changes rather fast with $E_{T}$ ) is sensitive to the relation between $E_{T}$ and impact parameter. We see from eq. (5) that this relation depends on the size of the contribution of the intra-nuclear cascade (parameter $k$ ). As mentioned above, the value $k=1 / 4000$ used here was obtained in [2] from the best fit of the correlation between $E_{T}$ and the energy $E_{Z D C}$ of the zero degree calorimeter. However, since we do not have a totally reliable expression for the latter, there is an uncertainty in the value of $k$. In order to illustrate its effect on $R\left(E_{T}\right)$, we show in Fig. 2 (dashed line) the result with $k=1 / 2000$, i.e. doubling the (comparatively small) contribution of the intra-nuclear cascade. The effect is concentrated in the lower half of the $E_{T}$ range. This uncertainty would not be present if the $J / \psi$ suppression were given as a function of either $E_{T}$ or charged multiplicity at mid-rapidities.

The large $E_{T}$ structure seen by the NA50 collaboration can also be explained assuming a deconfining phase transition [11]. At the energies of the Relativistic Heavy Ion Collider (RHIC) at Brookhaven, it will be possible to determine which of these two mechanisms is the correct one. Indeed, the transverse energy (or the corresponding energy density) where the structure has been seen by NA50, will be reached at RHIC well below 
the knee of the $E_{T}$ distribution, and, if our interpretation is correct, no structure will be present. It will, however, appear at higher values of $E_{T}$ - when reaching the knee of the $E_{T}$ distribution at $\sqrt{s}=200 \mathrm{GeV}$.

\section{ACKNOWLEGMENTS}

It is a pleasure to thank N. Armesto, C. Pajares, C. Gerschel, C. A. Salgado and Y. M. Shabelski for interesting discussions. We also thank B. Chaurand and M. Gonin for

providing numerical tables of the NA50 data. E. G. F. thanks Ministerio de Educacion y Cultura of Spain for financial support. 


\section{References}

[1] M. C. Abreu et al (NA50 collaboration), preprint CERN-EP-2000-013, to be published in Phys. Lett. B.

[2] N. Armesto, A. Capella and E. G. Ferreiro, Phys. Rev. C59 (1999).

[3] N. Armesto and A. Capella, Phys. Lett. B430, 23 (1998) ;

A. Capella, C. Gerschel and A. Kaidalov, Phys. Lett. B393, 431 (1997).

[4] D. Kharzeev, C. Lourenço, M. Nardi and H. Satz, Z. Phys. C74, 307 (1997).

[5] R. Vogt, Phys. Lett. B430, 15 (1998).

[6] J. Geiss et al, Phys. Lett. B447, 31 (1999) ;

J. Geiss et al, nucl-th 9810059 .

[7] C. Spieles et al, Phys. Rev. C60, 054901 (1999).

[8] D. E. Kahana and S. H. Kahana, Prog. Part. Nucl. Phys. 42, 269 (1999). See also nucl-th/9908063.

[9] J. Hüfner, Y. B. He and B. Z. Kopeliovich, hep-ph 9908244 ; A. Polleri, private communication.

[10] M. J. Leitch et al (E866/Nusea collaboration), nucl-exp 9909007.

[11] N. Armesto, M. A. Braun, E. G. Ferreiro and C. Pajares, Phys. Rev. Lett. 77, 3736 (1996) ;

M. Nardi and H. Satz, Phys. Lett. B442, 14 (1998);

D. Kharzeev, M. Nardi and H. Satz, hep-ph 9703308;

M. Nardi in Proceedings XI Rencontres de Blois, June 1999, Blois, France. 
J. Dias de Deus, R. Ugoccioni and R. Rodriguez, hep-ph/9907352.

For related work see J. P. Blaizot and J. Y. Ollitrault, Phys. Rev. Lett. 77, 1703 (1996) ; C. Y. Wang, Phys. Rev. C55, 2621 (1997) and ref. [4].

[12] M. C. Abreu et al (NA50 collaboration), Phys. Lett. B450, 456 (1999). 


\section{FIGURE CAPTIONS}

FIG. 1. The ratio $F\left(E_{T}\right)$ in eqs. (7), (8).

FIG. 2. The ratio $R\left(E_{T}\right)$ of $J / \psi$ over DY cross-sections, obtained with $\sigma_{a b s}=4.5 \mathrm{mb}$ and $\sigma_{c o}=1 \mathrm{mb}$, compared to the NA50 data [1] [12]. The full curve corresponds to $k=1 / 4000$ [2] in eq. (5). The dashed curve is obtained with $k=1 / 2000$ (see main text). The black points correspond to $1996 \mathrm{~Pb}-\mathrm{Pb}$ data, the black squares correspond to 1998 $\mathrm{Pb}-\mathrm{Pb}$ data, the white points to 1996 analysis with minimum bias and the white squares to 1998 analysis with minimum bias. 


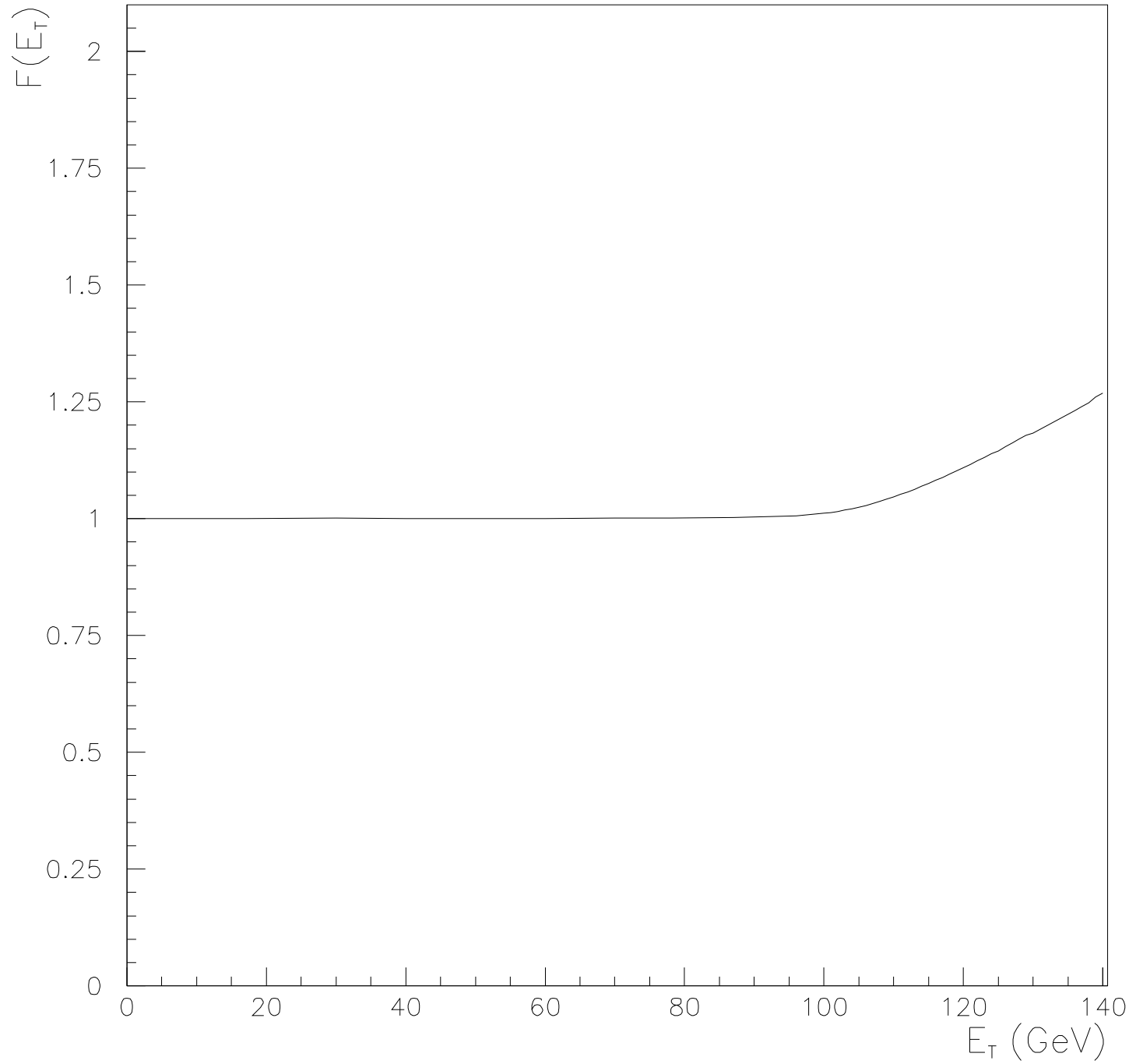

1 Capella 


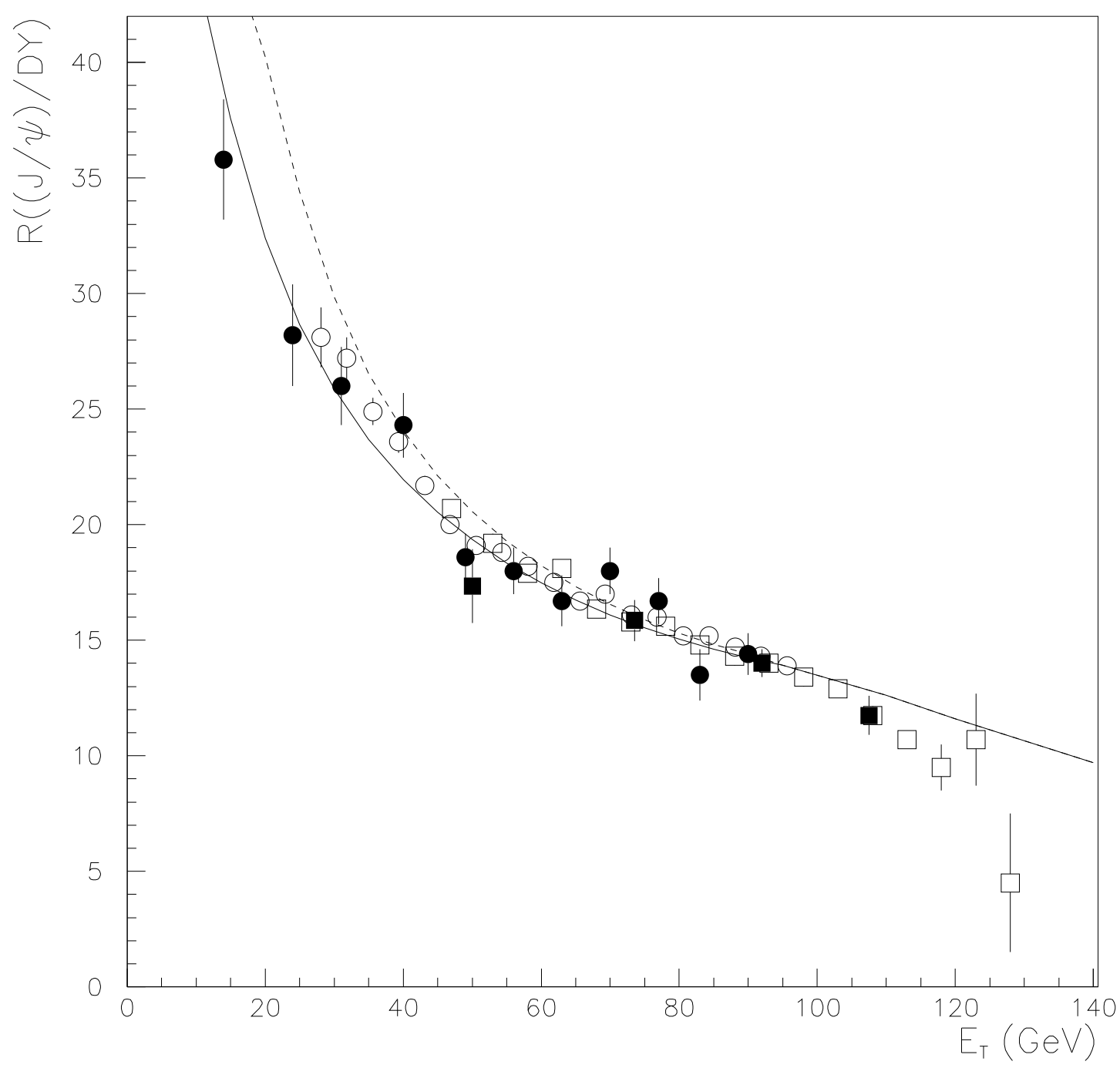

2 Capella 